Frequency of sleep advice sought by patients from community pharmacists $n=120$

Fifty-eight percent of CPs were 'very often' or 'often' asked through professional networks and social media. The survey asked CPs to record their perceptions on how often patients sought advice on several common sleep problems, as either 'very often' (most days), 'often' (1-3 times/week), 'occasionally' (1-3 times/month) or 'rarely' < once/month).

Other areas included self-rated confidence dealing with requests on sleep problems (1-5 Likert scale: 1 being 'not confident' and 5 'confident'), estimated proportion of GPreferred requests for advice and the perceived need for community-based sleep-related services. Data analyses were descriptive.

Results Completed responses were obtained from 120 CPs. Results summarised in figures 1 and 2. Combining 'often' and 'very often', the most common sleep-related problems encountered were insomnia (60\%) and sleepiness/tiredness/fatigue (54\%).

Half the CPs self-rated their confidence as 3 on the 'confident - not confident' scale. Fifty-seven percent reported that they received no undergraduate training on sleep problems. Proposed pharmacist involvement in sleep screening/signposting services or a pharmacy-based intervention/referral programme was supported by $78 \%$ and $70 \%$ respectively. about symptoms of OSAS, with the majority (93\%) asked 'occasionally'.

Discussion CPs frequently encounter patients with symptoms of sleep disorders, but their confidence and training in dealing with these is lacking. This study highlights the need for specialist sleep training for pharmacists and the development of pharmacy-based services to support and improve patient outcomes.

\title{
22 24-HOUR MOVEMENT BEHAVIOURS (PHYSICAL ACTIVITY, SEDENTARY BEHAVIOUR AND SLEEP) IMPACT ON ADOLESCENTS PHYSIOLOGICAL AND PSYCHOSOCIAL ADAPTATION TO TYPE 1 DIABETES: A MIXED METHODS SYSTEMATIC REVIEW
}

Mhairi Patience*, Xanne Janssen, Alison Kirk, Megan Crawford. University of Strathclyde, Glasgow, UK

10.1136/bmjresp-2021-bssconf.19 
Introduction 24-hour movement behaviours (physical activity, sedentary behaviour and sleep) are crucial for physical and mental health (Rollo, Antsygina, \& Tremblay, 2020). For adolescents with type 1 diabetes several unique challenges arise from navigating standard adolescent changes combined with the constant 24-hour management required for the condition (Cameron et al., 2018). Previous research has examined these behaviours in isolation, yet few have examined them relative to one another. The aim of this study was to conduct a mixed methods systematic review to comprehensively investigate 24-hour movement behaviours and their impact on glycemic control and quality of life in adolescents with type 1 diabetes.

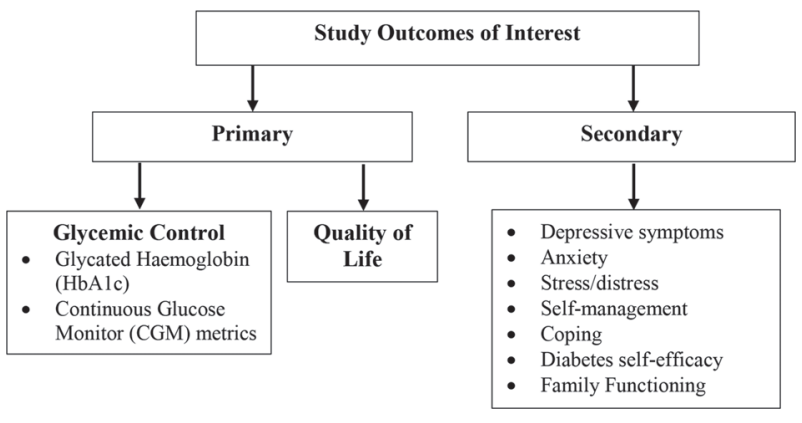

Abstract 22 Figure 1

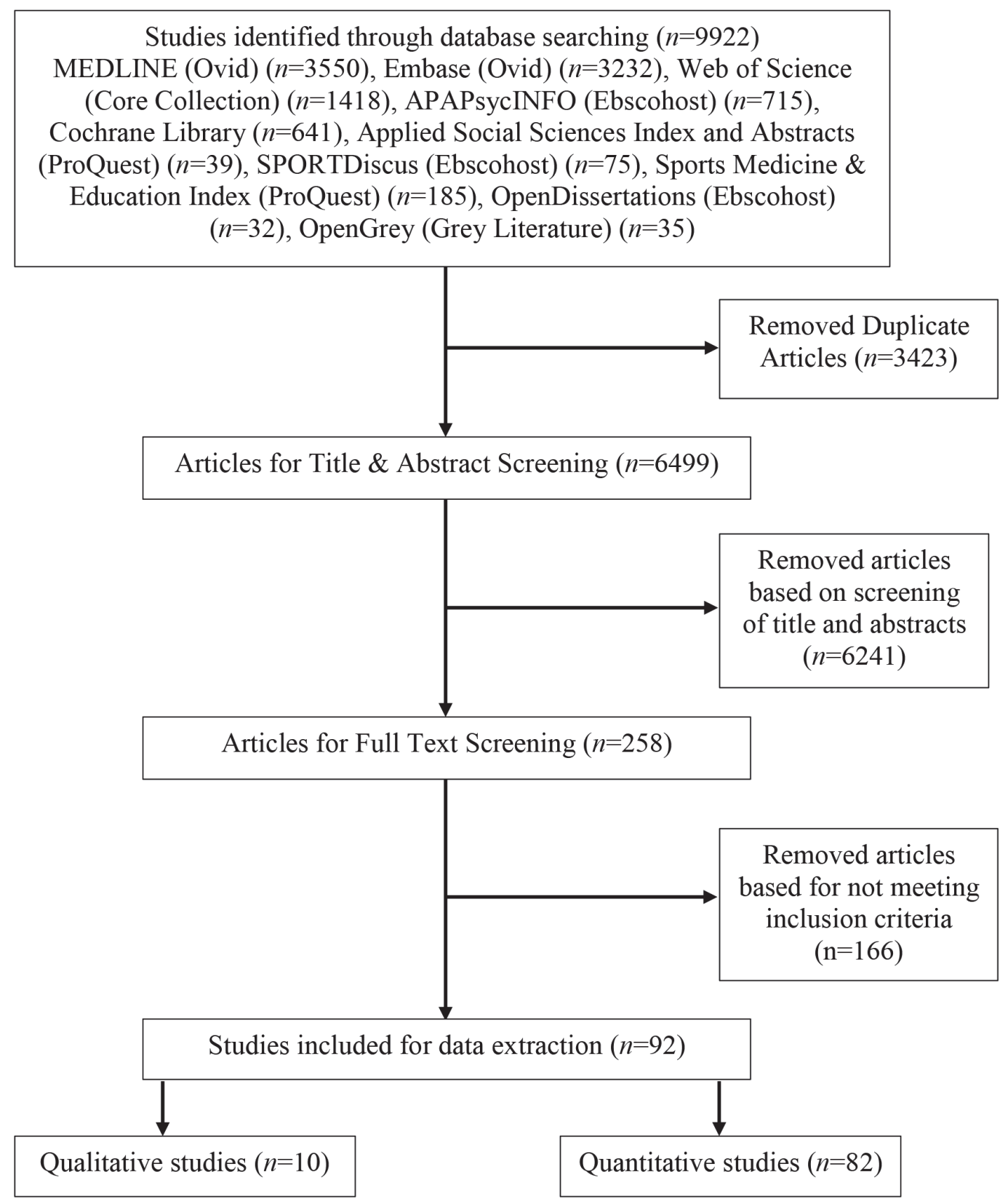

*Subject to change due to contacting of authors and planned reference review of included studies* 
Methods 10 databases were searched for quantitative and qualitative English language articles reporting at least one of the behaviours and their impact on priori primary and secondary outcomes (figure 1) in adolescents (11-18 years) with type 1 diabetes. There were no restrictions on article publication dates or study design. Articles were subjected to title and abstract screening, full text screening, data extraction and quality assessment.

Results In total 9922 articles were identified from the initial search with 92 articles included for data extraction after title, abstract and full text screening, (figure 2). Data analysis is ongoing, where possible a meta-analysis (quantitative), metaaggregation (qualitative) and mixed-methods synthesis (quantitative and qualitative narrative summary) will be conducted.

Discussion This extensive investigation on the full spectrum of 24-hour movement behaviours will identify the different, and perhaps complimentary, physiological and psychosocial impacts of each behaviour.

\section{REFERENCES}

1. Cameron FJ, Garvey K, Hood KK, Acerini CL, Codner E. ISPAD Clinical Practice Consensus Guidelines 2018: Diabetes in adolescence. Pediatr Diabetes 2018;19 Suppl 27:250-61.

2. Rollo S, Antsygina O, Tremblay MS. The whole day matters: Understanding 24hour movement guideline adherence and relationships with health indicators across the lifespan. J Sport Health Sci 2020.

\section{AHI DOES NOT ADEQUATELY REFLECT OSA SEVERITY}

Emma Lombard*, Simon Merritt. Conquest Hospital, East Sussex Healthcare NHS Trust, Hastings, UK

\subsection{6/bmjresp-2021-bssconf.20}

Introduction Obstructive sleep apnoea (OSA) results in significant cardiovascular consequences. Level of hypoxia and degree of sympathetic activation are postulated to play a role.

The Apnoea Hypopnoea Index (AHI) is used as a tool to assess severity of OSA. However, it does not measure depth or duration of hypoxia and may underestimate the risk of complications. The aim of this study was to evaluate the relationship between AHI and the burden of hypoxia.

Method This was a retrospective study, using data from noxturnal sleep studies. Equal numbers of each OSA severity, defined by AHI, were selected consecutively from 122 adult patients who underwent sleep studies between Dec 2020 and May 2021. Demographic data, AHI and percentage time spent with oxygen saturations $<90 \% \quad(\% \mathrm{~T}<90 \%)$ were recorded. Excel was used for analysis and Spearman's rank used to calculate the correlation coefficient (rho, r).

Result AHI was compared to $\% \mathrm{~T}<90 \%$ (figure 1) showing a moderate positive correlation (r0.6). Subgroup analysis demonstrated a moderate correlation in the severe group (r0.67), whereas only a very weak correlation in the moderate and mild groups (r0.19 and 0.16 respectively). There was no significant difference in the $\% \mathrm{~T}<90 \%$ in the moderate group compared to those with an AHI 30-60 (mean(SD) 14.86 (20.15) and 17.96(17.91) $\mathrm{P}=0.067)$ despite these patients having different categories of OSA severity.

Conclusion This study suggests that AHI inadequately reflects degree of hypoxic burden, and therefore is an incomplete measure of OSA disease severity. The results demonstrate patients with moderate OSA have a burden of hypoxia similar to many of those with severe disease. In these patients, AHI may inadequately reflect the risk of future complications

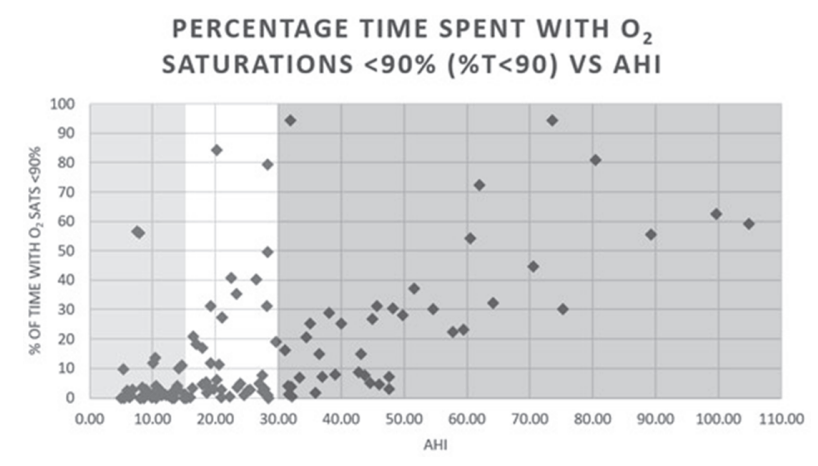

Abstract 23 Figure 1 Percentage time spent with $\mathrm{O}_{2}$ saturations $<90 \%(\% \mathrm{~T}<90)$ vs $\mathrm{AHI}$

resulting from hypoxia. Further research is needed to develop an alternate measure of severity to accurately reflect this risk, a composite of AHI and hypoxic burden would be a first step.

\section{\begin{tabular}{|l|l}
\hline 24 & INSOMNIA PREVALENCE IN CONFINED ELITE ATHLETES
\end{tabular}}

Rui Pereira*, Iuliana Hartescu, Luke Gupta, Robin C Jackson, Kevin Morgan. Loughborough University, Loughborough, UK

\subsection{6/bmjresp-2021-bssconf.21}

The demands of training, competition stress, and impact of frequent (inter)national travel are assumed to contribute to the shorter night-time sleep durations and poor overall sleep quality reported for elite athletes (1-3). However, systematically exploring this assumption is challenging, since a robust evaluation of sleep quality and practices in the presence and absence of sports participation would interrupt elite sports careers. The COVID-19 pandemic resulted in a cessation of international sport, and the home confinement of athletes. Using baseline data from an ongoing (pre-pandemic) study of athlete sleep, we compared the insomnia levels, sleep quantity and practices of elite athletes when exposed to, and deprived of

\section{Insomnia disorder categories}

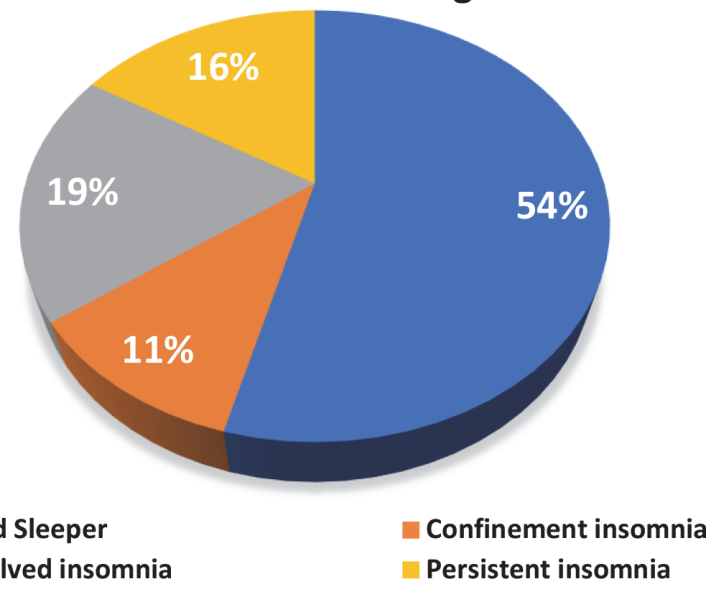

